\title{
Morphing quantum mechanics and fluid dynamics
}

\author{
Thomas Curtright $^{\S}$ and David Fairlie ${ }^{\otimes}$ \\ $\S$ Department of Physics, University of Miami, \\ Coral Gables, Florida 33124-8046, USA \\ ${ }^{\otimes}$ Department of Mathematical Sciences, University of Durham, \\ Durham, DH1 3LE, UK \\ Curtright@physics.miami.eduＤavid.Fairlie@durham.ac.uk
}

October 14, 2018

\begin{abstract}
We investigate the effects of given pressure gradients on hydrodynamic flow equations. We obtain results in terms of implicit solutions and also in the framework of an extra-dimensional formalism involving the diffusion/Schrödinger equation.
\end{abstract}

\section{Introduction}

There has recently been renewed interest in the similarities between the equations of fluid dynamics and those of quantum mechanics [1]-[8]. This is a venerable subject [9]. In a previous article [10] we have shown how any solution of the multi-variable, multi-dimensional generalization of the simplest flow equation, the Euler-Monge equation, is related to a particular type of solution to a linear diffusion or heat equation, in twice the number of "spatial" dimensions. But, as is well-known, the heat equation when complexified is just the Schrödinger equation. So the method of [10 may be viewed as a new transformation relating the Euler-Monge and dimensionally-doubled Schrödinger equations, which differs from the transformation of Madelung.

Here, we extend this result, especially in the case of one space dimension, to a flow equation driven by some particular, given pressure terms (i.e. so-called "body forces"). When the given pressure-gradient is either $t$ or $x$ independent, we obtain the general solution for such pressure driven flows in closed but implicit form, and we relate these solutions to those of driven diffusion/Schrödinger equations. The corresponding Schrödinger equation contains a potential whose form is related to the pressure-gradient. We also find explicit series solutions in a variety of situations. Special attention is given to constant and linear pressure-gradients. We discuss the complexification required to obtain the Schrödinger equation, and we emphasize analogies between the heat equation and the WignerMoyal equation for the evolution of quantum mechanical densities. In addition, we show how solutions of a related Bateman equation may be constructed.

Should there be any lack of motivation to consider such matters, perhaps a brief historical overview is warranted. The Euler-Monge (E-M) equations embodied in fluid dynamics first appeared in 18th and 19th century studies of both fluid flow [11] and analytic geometry [12. Riemann took up a study of the equations in the context of gas dynamics, discussing the equations as a theory of invariants [13] (for a modern textbook treatment, see [14]). His approach is widely applicable to almost all nonlinear flow problems, although it does not triumph over turbulence. A systematic modern discussion of the E-M equations that synthesizes ideas from both geometry and invariance theory can be found in the review by Dubrovin and Novikov [15. Most contemporary texts and reviews stress the universal role played by these nonlinear transport equations in accordance with Whitham's theory [16]: Essentially all nonlinear waves, even those in dispersive and dissipative media, involve E-M equations, or simple variants of them, if the nonlinear wavetrains are slowly varying. This makes the equations particularly useful for analyzing the asymptotic behavior of nonlinear solutions. The E-M equations and their conservation laws also serve as a useful starting point in Polyakov's study of turbulence [1] but without yet leading to a general solution of the Navier-Stokes equations. In short, the Euler-Monge equations appear widespread across a very broad landscape of physics and applied mathematics problems, and therefore it is important to understand their solutions at as many levels as possible. To that end we shall map some solutions of the E-M equations with given pressure-gradients onto linear diffusion/Schrödinger equations. 
Suppose we ask for the condition that $\mathbb{U}(x, t, a) \equiv \frac{1}{a} \exp a u(x, t)$ is a solution of the following linear diffusion equation in two ( $x$ and $a$ ) dimensions [17] with a given "potential" term $a \times g(x, t)$ :

$$
\left(\frac{\partial}{\partial t}-\frac{\partial^{2}}{\partial x \partial a}-a g(x, t)\right) \mathbb{U}(x, t, a)=0 .
$$

This holds if and only if a nonlinear equation is satisfied by $u=\frac{1}{a} \ln (a \mathbb{U})$ :

$$
\frac{\partial u(x, t)}{\partial t}=u(x, t) \frac{\partial u(x, t)}{\partial x}+g(x, t) .
$$

We will call this the "pressure driven Euler-Monge equation." The function $g$ is the pressure-gradient, $g(x, t)=$ $\partial_{x} p(x, t)$, as it would appear in the Navier-Stokes equation for an incompressible fluid without viscosity. Here, we shall concentrate on pressure-gradients which are given functions, especially those which are time-independent, $g(x)=\partial_{x} p(x)$, and not have $p$ determined by an equation of state. (Hence, our discussion is not truly a description of ideal non-viscous fluid flow.)

The linear equation (11) can always be attacked, at least formally, by the propagator method. However, in general, it is not possible to find explicit, closed-form solutions, either to that linear equation or to the associated nonlinear equation (2). Nevertheless, general implicit solutions to the nonlinear equation can be obtained in closed form, as shown below, when the pressure-gradient is either a function of $x$ alone, or of $t$ alone. Moreover, in cases where the pressure takes simple forms, special explicit solutions exist, in terms of which the general solution may be constructed.

\section{Simple examples}

In this section we discuss some simple cases before treating a general pressure term. The technique we use is a variant of the well-known method of characteristics.

First, consider the case of the constant pressure equation, with $g(x)=0$.

$$
\frac{\partial u}{\partial t}=u \frac{\partial u}{\partial x}
$$

Two elementary special solutions are given by

$$
x+u t=0, \quad u=0 .
$$

Now, to obtain the general solution, just set one of these expressions to be an arbitrary differentiable function of the other.

$$
x+u t=G(u),
$$

for any differentiable $G$, where in (5), $u=u(x, t)$. This result is well-known [16. By taking $x$ and $t$ partial derivatives, the reader will easily verify that as a consequence of (515), $u(x, t)$ must satisfy the Euler-Monge equation (3).

Next, consider the equation resulting from a linear pressure, $p(x)=p(0)+k x$, hence constant pressure-gradient, $g(x)=k$.

$$
\frac{\partial u}{\partial t}=u \frac{\partial u}{\partial x}+k
$$

Two special solutions are given by

$$
x+u t-\frac{1}{2} k t^{2}=0, \quad u-k t=0 .
$$

Then the general solution is given by

$$
x+u t-\frac{1}{2} k t^{2}=G(u-k t),
$$

where again $G$ is any differentiable function, and where $u=u(x, t)$ in this last equation.

In the case of a quadratic pressure the equation becomes

$$
\frac{\partial u}{\partial t}=u \frac{\partial u}{\partial x}+k^{2} x,
$$


with a linear forcing term $k^{2} x$. A particular solution is now given by

$$
k x \cos (k t)+u \sin (k t)=0 .
$$

(Dividing by $k$ and taking the limit $k \rightarrow 0$ recovers the first of (4).) Now we can construct another solution by translating $k t$ by $\frac{\pi}{2}$ to give

$$
k x \sin (k t)-u \cos (k t)=0 .
$$

(In the $k \rightarrow 0$ limit this gives the trivial solution in (44).) The general solution is now given implicitly by

$$
k x \cos (k t)+u(x, t) \sin (k t)=G(k x \sin (k t)-u(x, t) \cos (k t)),
$$

with $G$ an arbitrary differentiable function.

\section{Recipe for a general implicit solution}

One way of proceeding with equation (2), in the case of arbitrary time-independent $g(x)$, is not to consider $u$ as a function of $x$ and $t$, but to think of $t$ as a function of $x$ and $u$, i.e. the hodograph method [16]. Then we have

$$
\frac{\partial u}{\partial x}=-\frac{\partial t(x, u) / \partial x}{\partial t(x, u) / \partial u}, \quad \frac{\partial u}{\partial t}=\frac{1}{\partial t(x, u) / \partial u}
$$

This transforms equation (2) into a linear equation for $t$.

$$
\frac{\partial}{\partial x}(u t(x, u)+x)=g(x) \frac{\partial}{\partial u} t(x, u) .
$$

The solution for general $g(x)$ is easily constructed from this. We turn this equation into an integro-differential equation by integration with respect to $x$.

$$
t(x, u)=F(u)-\frac{x}{u}+\frac{1}{u} \frac{\partial}{\partial u} \int_{0}^{x} t(z, u) g(z) d z,
$$

where $F(u)$ is an arbitrary function of integration. This equation is then formally solved by iteration:

$$
\begin{aligned}
t(x, u)= & F(u)-\frac{x}{u}+\frac{1}{u} \frac{\partial}{\partial u} \int_{0}^{x} d z_{1} g\left(z_{1}\right)\left(F(u)-\frac{z_{1}}{u}\right)+\frac{1}{u} \frac{\partial}{\partial u} \int_{0}^{x} d z_{1} g\left(z_{1}\right) \frac{1}{u} \frac{\partial}{\partial u} \int_{0}^{z_{1}} d z_{2} g\left(z_{2}\right) t\left(z_{2}, u\right) \\
& =F(u)-\frac{x}{u}+\cdots+\int_{0}^{x} d z_{1} g\left(z_{1}\right) \cdots \int_{0}^{z_{n-1}} d z_{n} g\left(z_{n}\right)\left(\frac{1}{u} \frac{\partial}{\partial u}\right)^{n}\left(F(u)-\frac{z_{n}}{u}\right)+\cdots .
\end{aligned}
$$

Since every term in the expansion is a product of a function of $x$ with a function of $u$, all these terms are easily disentangled. The terms linear in $F$ can be evaluated separately to give

$$
\begin{aligned}
& \left(1+\int_{0}^{x} d z_{1} g\left(z_{1}\right) \frac{1}{u} \frac{\partial}{\partial u}+\int_{0}^{x} d z_{1} g\left(z_{1}\right) \int_{0}^{z_{1}} d z_{2} g\left(z_{2}\right)\left(\frac{1}{u} \frac{\partial}{\partial u}\right)^{2}+\cdots\right) F(u) \\
& =\sum_{n=0}^{\infty} \frac{1}{n !}\left(\int_{0}^{x} d z g(z)\right)^{n}\left(\frac{1}{u} \frac{\partial}{\partial u}\right)^{n} F(u)=e^{\int_{0}^{x} d z g(z) \frac{1}{u} \frac{\partial}{\partial u}} F(u)=F\left(u \sqrt{1+\frac{2}{u^{2}} \int_{0}^{x} d z g(z)}\right) .
\end{aligned}
$$

The $(n+1)$ th term in the remaining series of terms is given by $(-1)^{n+1}(2 n-1) ! ! / u^{2 n+1}$ times

$$
\int_{0}^{x} d z_{1} g\left(z_{1}\right) \int_{0}^{z_{1}} d z_{2} g\left(z_{2}\right) \cdots \int_{0}^{z_{n-1}} d z_{n} g\left(z_{n}\right) z_{n}=\frac{1}{(n-1) !} \int_{0}^{x}(p(x)-p(z))^{n-1} g(z) z d z
$$

where the pressure is $p(x)=p(0)+\int_{0}^{x} d z g(z)$. These terms can be summed. After an integration by parts, the final result is

$$
t(x, u)=F\left(u \sqrt{1+\frac{2}{u^{2}}(p(x)-p(0))}\right)-\frac{1}{u} \int_{0}^{x} \frac{d z}{\sqrt{1+\frac{2}{u^{2}}(p(x)-p(z))}} .
$$

This is the general solution to (14) with boundary value $t(x=0, u)=F(u)$. 
The result (19) is easily checked to be a solution to (14). We recognize this as again being of the form where an arbitrary function of one particular solution of (2) is set equal to a second particular solution. In this case $F\left(u \sqrt{1+\frac{2}{u^{2}}(p(x)-p(0))}\right) \equiv G\left(\frac{1}{2} u^{2}+p(x)\right)$ involves an arbitrary function of the combination that gives a particular time-independent solution, $\frac{1}{2} u^{2}+p(x)=$ constant, of the time-independent special case of (2), $\frac{1}{2} \partial_{x}\left(u^{2}\right)=-\partial_{x} p(x)$, and the remaining terms in $t(x, u)$ implicitly furnish another particular solution of the fully time-dependent (2).

Similar statements apply to the situation where the driving term is a function of only $t$, and not $x$. Thus $\partial u / \partial t=u \partial u / \partial x+k(t)$ is solved implicitly by $x+u t-\int_{0}^{t} k(\tau) \tau d \tau=G\left(u-\int_{0}^{t} k(\tau) d \tau\right)$, where the latter function has as its argument the same form as the particular $x$-independent solution $u-\int_{0}^{t} k(\tau) d \tau=$ constant.

\section{Series solutions}

Building up to the extra dimension Of course, the existence of an implicit solution, while providing a pleasing theoretical establishment of integrability, is not much use in many practical applications. So we also look for power series expansions in $t$. Consider first the case of a constant pressure-gradient,

$$
\frac{\partial u}{\partial t}=u \frac{\partial u}{\partial x}+k
$$

Further time differentiation removes the explicit $k$, just as it would for any time-independent $g(x)$,

$$
\frac{\partial^{2} u}{\partial t^{2}}=\frac{1}{2} \frac{\partial}{\partial t} \frac{\partial}{\partial x} u^{2}=\frac{\partial}{\partial t}\left(u \frac{\partial u}{\partial x}\right)=\frac{\partial}{\partial x}\left(u \frac{\partial u}{\partial t}\right) .
$$

If the power series is denoted by

$$
u(x, t)=\sum_{n=0}^{\infty} t^{n} u_{n}(x),
$$

we have $u(x, 0) \equiv u(x)=u_{0}, u_{1}=k+\frac{1}{2} \frac{\partial}{\partial x} u(x)^{2}$, and so on. That is,

$$
(n+1) u_{n+1}=\frac{1}{2} \frac{\partial}{\partial x} \sum_{j=0}^{n} u_{j} u_{n-j}, \text { for } n \geq 1 .
$$

The resulting expression for $\partial^{n} u /\left.\partial t^{n}\right|_{t=0}=n ! u_{n}$ for $n \geq 2$ is therefore given, for constant $k$, by

$$
\left.\frac{\partial^{n} u}{\partial t^{n}}\right|_{t=0}=\sum_{j=0}^{\lfloor n / 2\rfloor}\left(\frac{k}{2}\right)^{j} \frac{n !}{j !(n-2 j) !} \frac{\partial^{n-j-1}}{\partial x^{n-j-1}}\left(u(x)^{n-2 j} \frac{\partial u(x)}{\partial x}\right) .
$$

Here, the floor function, $\lfloor n / 2\rfloor$, is the greatest integer less than or equal to $n / 2$. Hence,

$$
\begin{aligned}
u(x, t) & =u(x)+k t+\sum_{n=1}^{\infty} t^{n} \sum_{j=0}^{\lfloor n / 2\rfloor} \frac{1}{j !(n-2 j+1) !}\left(\frac{k}{2}\right)^{j} \frac{\partial^{n-j}}{\partial x^{n-j}} u(x)^{n-2 j+1} \\
& =u(x)+k t+\left.\sum_{n=1}^{\infty} t^{n} \sum_{j=0}^{\lfloor n / 2\rfloor} \frac{1}{j !(n-2 j+1) !}\left(\frac{k}{2} \frac{\partial}{\partial x}\right)^{j}\left(\frac{\partial^{2}}{\partial a \partial x}\right)^{n-2 j} e^{a u(x)} u(x)\right|_{a=0} .
\end{aligned}
$$

This last expression involving the exponential, evaluated at $a=0$ after all differentiations, evokes the extradimensional approach.

To follow through on that idea, recall that Hermite polynomials are given by $(18, \mathbf{2 2 . 3 . 1 0})$

$$
H_{n}(z)=n ! \sum_{j=0}^{\lfloor n / 2\rfloor} \frac{1}{j !(n-2 j) !}(-1)^{j}(2 z)^{n-2 j}=(2 z)^{n} n ! \sum_{j=0}^{\lfloor n / 2\rfloor} \frac{1}{j !(n-2 j) !}\left(\frac{-1}{4 z^{2}}\right)^{j} .
$$


So, taking $\partial / \partial x$ of both sides of (24), we find, formally,

$$
\begin{gathered}
\left.\frac{\partial^{n}}{\partial t^{n}} \frac{\partial}{\partial x} u\right|_{t=0}=\sum_{j=0}^{\lfloor n / 2\rfloor} \frac{(k / 2)^{j}}{(j) !(n-2 j) !} \frac{\partial^{n-j}}{\partial x^{n-j}}\left(u^{n-2 j} u_{x}\right)=\left.\sum_{j=0}^{\lfloor n / 2\rfloor} \frac{1}{(j) !(n-2 j) !}\left(\frac{k}{2} \frac{\partial}{\partial x}\right)^{j}\left(\frac{\partial^{2}}{\partial x \partial a}\right)^{n-2 j} e^{a u} u_{x}\right|_{a=0} \\
=\left.\left(\frac{1}{\sqrt{-1}} \sqrt{\frac{k}{2} \frac{\partial}{\partial x}}\right)^{n} \sum_{j=0}^{\lfloor n / 2\rfloor} \frac{1}{(j) !(n-2 j) !}(-1)^{j}\left(\frac{\sqrt{-1} \frac{\partial^{2}}{\partial x \partial a}}{\sqrt{\frac{k}{2} \frac{\partial}{\partial x}}}\right)^{n-2 j} e^{a u} u_{x}\right|_{a=0} \\
=\left.\left(\frac{1}{\sqrt{-1}} \sqrt{\frac{k}{2} \frac{\partial}{\partial x}}\right)^{n} \frac{1}{n !} H_{n}\left(\frac{\sqrt{-1} \frac{\partial^{2}}{\partial \sqrt{k} a}}{2 \sqrt{\frac{k}{2}} \frac{\partial}{\partial x}}\right) e^{a u} u_{x}\right|_{a=0} .
\end{gathered}
$$

This result allows us to perform the sum, to obtain

$$
\begin{aligned}
\frac{\partial}{\partial x} u(x, t) & =\sum_{n=0}^{\infty} t^{n} \frac{\partial}{\partial x} u_{n}=\left.\sum_{n=0}^{\infty}\left(\frac{t}{\sqrt{-1}} \sqrt{\frac{k}{2} \frac{\partial}{\partial x}}\right)^{n} \frac{1}{n !} H_{n}\left(\frac{\sqrt{-1} \frac{\partial^{2}}{\partial x \partial a}}{2 \sqrt{\frac{k}{2} \frac{\partial}{\partial x}}}\right) e^{a u(x)} u_{x}(x)\right|_{a=0} \\
& =\left.\exp \left(\frac{2 t}{\sqrt{-1}} \sqrt{\frac{k}{2} \frac{\partial}{\partial x}} \frac{\sqrt{-1} \frac{\partial^{2}}{2 x \partial a}}{2 \sqrt{\frac{k}{2} \frac{\partial}{\partial x}}}-\left(\frac{t}{\sqrt{-1}} \sqrt{\frac{k}{2} \frac{\partial}{\partial x}}\right)^{2}\right) e^{a u(x)} u_{x}(x)\right|_{a=0} \\
& =\left.\exp \left(t \frac{\partial^{2}}{\partial x \partial a}+\frac{1}{2} k t^{2} \frac{\partial}{\partial x}\right) e^{a u(x)} u_{x}(x)\right|_{a=0} \\
& =\left.e^{t \frac{\partial^{2}}{\partial x \partial a}+\frac{1}{2} k t^{2} \frac{\partial}{\partial x}} \frac{\partial}{\partial x} \frac{e^{a u(x)}-1}{a}\right|_{a=0},
\end{aligned}
$$

through use of the generating function $\left([18, \mathbf{2 2 . 9 . 1 7}) e^{2 z s-s^{2}}=\sum_{n=0}^{\infty} \frac{1}{n !} s^{n} H_{n}(z)\right.$. Thus we conclude

$$
u(x, t)=k t+\left.e^{t \frac{\partial^{2}}{\partial x \partial a}+\frac{1}{2} k t^{2} \frac{\partial}{\partial x}}\left(\frac{e^{a u(x)}-1}{a}\right)\right|_{a=0}=k t+\left.e^{t \frac{\partial^{2}}{\partial x \partial a}} \frac{1}{a}\left(e^{a u\left(x+\frac{1}{2} k t^{2}\right)}-1\right)\right|_{a=0} .
$$

The RHS clearly involves just the time evolution of $\mathbb{U}$, evaluated at the extra dimension boundary, $a=0$, but under the action of a slightly modified extension of the time-dependent kernel in [10, such that the initial data has a supplementary shift $x \rightarrow x+\frac{1}{2} k t^{2}$.

Comparing this to the undriven situation, we see the physical interpretation is simply that of a constant external acceleration imposed uniformly on the nonlinear self-interaction of the fluid. If we "fall" along with the selfinteracting fluid, constantly accelerating with it, the effects of the external acceleration are removed. That is to say, from Galilean covariance, if $u(x, t)$ is any solution of (3), then $u\left(x+\frac{1}{2} k t^{2}, t\right)+k t$ is a solution of (66).

Working down from the extra dimension The previous implicit solutions suggest another way to obtain results in terms of an extra-dimensional equation: Incorporate one of the particular solutions into the exponential along with $u(x, t)$. For example, define

$$
\mathfrak{U}(x, t, a)=\frac{1}{a}\left(e^{a u(x, t)-a k x \tan (k t)}-1\right) .
$$

Then we have

$$
\left(\frac{\partial}{\partial t}-\frac{\partial^{2}}{\partial a \partial x}-k \tan k t\left(1+x \frac{\partial}{\partial x}+a \frac{\partial}{\partial a}\right)\right) \mathfrak{U}(x, t, a)=e^{a u(x, t)-a k x \tan (k t)}\left(\partial_{t} u(x, t)-u(x, t) \partial_{x} u(x, t)-k^{2} x\right) .
$$

This gives an equivalence between the Euler-Monge equation with linear pressure-gradients, (9), and solutions, of the form given in (30), to the following modified extra-dimensional linear equation.

$$
\left(\frac{\partial}{\partial t}-\frac{\partial^{2}}{\partial a \partial x}\right) \mathfrak{U}(x, t, a)=(k \tan k t)\left(1+x \frac{\partial}{\partial x}+a \frac{\partial}{\partial a}\right) \mathfrak{U}(x, t, a) .
$$

The formal solution to this linear equation is given by the propagator method.

$$
\mathfrak{U}(x, t, a)=\mathbf{K}(t) \mathfrak{U}(x, t=0, a)=\mathbf{K}(t) \frac{1}{a}\left(e^{a u(x)}-1\right),
$$

where the operator kernel obeys

$$
\frac{\partial}{\partial t} \mathbf{K}(t)=\left(\frac{\partial^{2}}{\partial x \partial a}+(k \tan k t)\left(1+x \frac{\partial}{\partial x}+a \frac{\partial}{\partial a}\right)\right) \mathbf{K}(t) .
$$


That is, the kernel is a time-ordered exponential [19] given by the usual series of nested integrals.

$$
\begin{aligned}
& \mathbf{K}(t)=1+\sum_{j=1}^{\infty} \int_{0}^{t} d \tau_{1} \int_{0}^{\tau_{1}} d \tau_{2} \cdots \int_{0}^{\tau_{j-1}} d \tau_{j}\left(\frac{\partial^{2}}{\partial x \partial a}+k \tan k \tau_{1}\left(1+x \frac{\partial}{\partial x}+a \frac{\partial}{\partial a}\right)\right) \times \\
& \times\left(\frac{\partial^{2}}{\partial x \partial a}+k \tan k \tau_{2}\left(1+x \frac{\partial}{\partial x}+a \frac{\partial}{\partial a}\right)\right) \cdots\left(\frac{\partial^{2}}{\partial x \partial a}+k \tan k \tau_{j}\left(1+x \frac{\partial}{\partial x}+a \frac{\partial}{\partial a}\right)\right) .
\end{aligned}
$$

This time-ordered exponential can be worked out as products of ordinary exponentials, through use of the underlying algebra.

$$
[A, C]=0=[B, C], \quad[A, B]=A
$$

where $A=\frac{\partial^{2}}{\partial x \partial a}, B=a \frac{\partial}{\partial a}, C=1+x \frac{\partial}{\partial x}-a \frac{\partial}{\partial a}$. Note that $C$ is central. Writing

$$
\begin{aligned}
\frac{\partial^{2}}{\partial x \partial a}+(k \tan k t)\left(1+x \frac{\partial}{\partial x}+a \frac{\partial}{\partial a}\right) & =\frac{\partial^{2}}{\partial x \partial a}+2(k \tan k t)\left(a \frac{\partial}{\partial a}\right)+(k \tan k t)\left(1+x \frac{\partial}{\partial x}-a \frac{\partial}{\partial a}\right) \\
& =A+2(k \tan k t) B+(k \tan k t) C
\end{aligned}
$$

we see that we need to solve an evolution equation of the form

$$
\frac{d}{d t} \mathbf{K}(t)=(\alpha(t) A+\beta(t) B+\gamma(t) C) \mathbf{K}(t)
$$

with $\mathbf{K}(t=0)=1$, where $A, B, C$ obey (36), and where the coefficients $\alpha, \beta, \gamma$ commute with everything. The solution is given by the Baker-Campbell-Hausdorff technique.

$$
\begin{aligned}
\mathbf{K}(t) & =\exp \left(B \int_{0}^{t} \beta(\tau) d \tau\right) \exp \left(A \int_{0}^{t} \alpha(\tau) e^{\int_{0}^{\tau} \beta\left(\tau^{\prime}\right) d \tau^{\prime}} d \tau\right) \exp \left(C \int_{0}^{t} \gamma(\tau) d \tau\right) \\
& =\exp \left(A \int_{0}^{t} \alpha(\tau) e^{-\int_{\tau}^{t} \beta\left(\tau^{\prime}\right) d \tau^{\prime}} d \tau\right) \exp \left(B \int_{0}^{t} \beta(\tau) d \tau\right) \exp \left(C \int_{0}^{t} \gamma(\tau) d \tau\right)
\end{aligned}
$$

For the case at hand, $\alpha(t)=1, \beta(t)=2 k \tan k t=2 \gamma(t), \int_{0}^{t} \beta(\tau) d \tau=-\ln \cos ^{2} k t=2 \int_{0}^{t} \gamma(\tau) d \tau$, and $\int_{0}^{t} \alpha(\tau) e^{-\int_{\tau}^{t} \beta\left(\tau^{\prime}\right) d \tau^{\prime}} d \tau=\frac{1}{2 k} \sin 2 k t$. So

$$
\begin{aligned}
& \mathbf{K}(t)=\exp \left(\frac{1}{2 k} A \sin (2 k t)\right) \exp \left(-B \ln \cos ^{2} k t\right) \exp \left(-\frac{1}{2} C \ln \cos ^{2} k t\right) \\
= & \frac{1}{\cos k t} \exp \left(\frac{1}{2 k} \sin (2 k t) \frac{\partial^{2}}{\partial x \partial a}\right) \exp \left(-\frac{1}{2} \ln \left(\cos ^{2} k t\right)\left(x \frac{\partial}{\partial x}+a \frac{\partial}{\partial a}\right)\right) .
\end{aligned}
$$

The right-most exponential operator rescales $x$ and $a$ in the initial data, and the left-most operator then evolves these data as in the undriven case, except for the replacement $t \rightarrow \frac{1}{2 k} \sin (2 k t)$.

Thus the action of this kernel on the initial, exponentiated data becomes

$$
\mathfrak{U}(x, t, a)=\mathbf{K}(t)\left(\frac{e^{a u(x)}-1}{a}\right)=e^{\frac{1}{2 k} \sin (2 k t) \frac{\partial^{2}}{\partial x \partial a}}\left(\frac{e^{u(x / \cos k t) a / \cos k t}-1}{a}\right) .
$$

As in [10, series expansion in powers of $a$ and evaluation of (41) at $a=0$ produces a time series solution for $u(x, t)$, since $\lim _{a \rightarrow 0} \mathfrak{U}(x, t, a)=\lim _{a \rightarrow 0} \frac{1}{a}\left(e^{a u(x, t)-a k x \tan (k t)}-1\right)=u(x, t)-k x \tan (k t)$. The series so obtained explicitly exhibits any difference between $u$ and the particular solution incorporated into the exponential.

$$
u(x, t)=k x \tan (k t)+\frac{1}{\cos k t} \sum_{j=0}^{\infty} \frac{1}{(1+j) !}\left(\frac{1}{k} \sin k t\right)^{j} \frac{d^{j}}{d x^{j}}\left(u\left(\frac{x}{\cos k t}\right)\right)^{1+j} .
$$

Once having found these results so systematically - although perhaps somewhat laboriously - their interpretation is almost as simple as for the case of a constant driving term. As the reader may check by direct substitution, if $u(x, t)$ is any solution of (3), then $\frac{1}{\cos k t} u\left(\frac{x}{\cos k t}, \frac{1}{k} \tan k t\right)+k x \tan k t$ is a solution of (9) resulting from the same initial value data [20, 21, 22]. 
The preceding modification of the propagator approach to (1) can be employed for general pressure-gradients. If $u_{p}(x, t)$ is a particular solution to (2), even if the pressure-gradient depends on both $x$ and $t$, then

$$
\mathfrak{U}(x, t, a)=\frac{1}{a}\left(e^{a u(x, t)-a u_{p}(x, t)}-1\right)
$$

satisfies the linear equation

$$
\left(\frac{\partial}{\partial t}-\frac{\partial^{2}}{\partial a \partial x}-\left(\partial_{x} u_{p}(x, t)\right)\left(1+a \frac{\partial}{\partial a}\right)-u_{p}(x, t) \frac{\partial}{\partial x}\right) \mathfrak{U}(x, t, a)=0
$$

if and only if $\partial u(x, t) / \partial t=u(x, t) \partial u(x, t) / \partial x+g(x, t)$. Thus, evolution of any exponentiated initial data, expressed as $\exp \left(u(x)-u_{p}(x)\right)$, can be effected by solving a linear equation for the kernel appropriate to (44), and acting with that kernel on $\mathfrak{U}(x, t=0, a)$. For certain problems, in addition to the $g(x)=k^{2} x$ case just discussed, this approach may be simpler to carry through than a direct attempt to construct the propagator for (11).

Breaking waves, shocks, and all that The formation of shocks under Euler-Monge evolution is well-known, and has a simple qualitative description in terms of characteristics (for example, see [14, 16]). It is perhaps not so well-known that the series solution (25) leads to the same conclusions. In general, this may require some asymptotic approximations, but it is easy to find simple analytical examples.

A particularly elementary illustration of a breaking kinematic wave is given by taking continuous, piecewise linear $u$ profiles. First consider the case of the undriven Euler-Monge equation. The time power series sum (25) for $k=0$ is easily evaluated in this case. Take one particular initial linear segment to be

$$
u(x)=\alpha+\beta x .
$$

Then we have $\frac{1}{(n+1) !} \frac{\partial^{n}}{\partial x^{n}} u(x)^{n+1}=\beta^{n}(\alpha+\beta x)$, and so, for the time development of this segment,

$$
u(x, t)=(\alpha+\beta x)\left(1+\sum_{n=1}^{\infty}(\beta t)^{n}\right)=\frac{\alpha+\beta x}{1-\beta t} .
$$

The segment becomes vertical at its "break-time" $t_{\text {break }}=1 / \beta$, and while obvious, it is perhaps worth stressing that this is precisely the same as the radius of convergence of the time power series (25).

Continuously rising and falling combinations of such linear segments, forming a triangular "bump," with equal amounts of rise and fall, have conserved height and base length. Hence the triangle's area is constant. So too for the area under a continuous polygonal bump. This area conservation is just what you would expect from the Euler-Monge equation, written as a local conservation law: $\partial_{t} u(x, t)=\partial_{x}\left(u^{2}(x, t) / 2\right)$. At least, this is what you would expect for continuous and differentiable functions. The fact that the same integrated conservation law also applies to continuous, piecewise differentiable functions may be surprising upon first encounter, but it is well-known to be true 14, 16.

After the earliest such break-time, the continuous piecewise linear $u$ profile will no longer be single-valued. If there are physical reasons to prohibit this, a standard procedure is to replace the continuous but multi-valued $u$ with a shock discontinuity, positioned so as to preserve the previously mentioned area conservation (cf. the RankineHugoniot rule, or the Lax entropy condition 23]).

When constant pressure-gradients drive the Euler-Monge equation, the result (25) shows the same initial linear segment evolves as

$$
u(x, t)=k t+\frac{\alpha+\beta\left(x+\frac{1}{2} k t^{2}\right)}{1-\beta t},
$$

with the same break-time as before, $t_{\mathrm{break}}=1 / \beta$. When linear pressure-gradients are added to the Euler-Monge equation, (42) shows the initial linear segment evolves as

$$
u(x, t)=k x \tan k t+\frac{\alpha+\beta x / \cos k t}{\cos k t-\frac{\beta}{k} \sin k t},
$$

with modified break-time given by $t_{\text {break }}=\frac{1}{k} \arctan (k / \beta)$. Again, these break-times are just the radii of convergence for the respective time power series. 
Another example is given by taking exponential initial data, for which we take the $k=0$ case to again see the wave evolution from the series (25). Suppose that initially $u(x)=A e^{x / L}$, then $\frac{\partial^{n}}{\partial x^{n}} u(x)^{n+1}=\left(\frac{n+1}{L}\right)^{n}\left(A e^{x / L}\right)^{n+1}$, so

$$
u(x, t)=A e^{x / L}\left(1+\sum_{n=1}^{\infty} \frac{(n+1)^{n}}{(n+1) !}\left(\frac{A t}{L} e^{x / L}\right)^{n}\right)=\frac{L}{t} \sum_{n=1}^{\infty} \frac{n^{n-1}}{n !}\left(\frac{A t}{L} e^{x / L}\right)^{n} .
$$

This series is familiar to anyone who has ever attempted to find by iteration the inverse function for the elementary equation $x(y)=y e^{y}$, i.e. to obtain $y(x)=x e^{-y}=x-x^{2}+\frac{3}{2} x^{3}-\frac{8}{3} x^{4}+-\cdots=x \sum_{n=1}^{\infty}(-n x)^{n-1} / n$ !. Lambert and Euler studied the analytic properties of a generalization. There is a contemporary movement to standardize the function and name it after Lambert [24]. The resulting "Lambert W function" series definition, its log derivative, and its key functional relation are given by

$$
W(z)=-\sum_{n=1}^{\infty} \frac{1}{n !} n^{n-1}(-z)^{n}, \quad z \frac{d}{d z} W(z)=\frac{W(z)}{1+W(z)}, \quad W(z) \exp (W(z))=z .
$$

This series converges absolutely for $|z|<1 / e$.

So the above solution (49) for exponential initial data is

$$
u(x, t)=-\frac{L}{t} W\left(-\frac{A t}{L} e^{x / L}\right) .
$$

The radius of convergence of the series for $u$ is therefore both $t$ - and $x$-dependent. Absolute convergence holds when $\frac{A t}{L} e^{x / L}<1 / e$. Actually, the Lambert function is well-behaved for all positive arguments, as is obvious from the inverse function, hence the exponential shape evolves backward in time without the formation of any singularities. But for forward time evolution, the wave always breaks, or forms a shock-front, and it does so at earlier times for larger $x$.

The condition for the wave to break, for positive $t$, is just that the radius of convergence of the time power series is reached. That is $t_{\text {break }}=\frac{L}{A} e^{-1-x / L}$. At this time, for the given position, the slope of the wave profile becomes vertical, since

$$
L \frac{d}{d x} u(x, t)=\frac{u(x, t)}{1-t u(x, t) / L},
$$

and $W(-1 / e)=-1$ gives $u\left(x, t=\frac{L}{A} e^{-1-x / L}\right)=L / t$. The motion of the vertical face of the wave is simply given by $\frac{A t}{L} e^{x / L}=\frac{1}{e}$ or

$$
x(t)=L\left(\ln \frac{L}{A}-1-\ln t\right) .
$$

There is also the implicit definition of $u(x, t)$ provided by (5) when specialized to the case at hand: $x+u t=G(u)=$ $L \ln (u / A)$, or

$$
u(x, t)=A e^{x / L} \exp (t u(x, t) / L) .
$$

This functional relation is immediately seen to be the familiar one obeyed by the Lambert function, as in (150) above. Indeed, our derivation of the series solution to the Euler-Monge equation for general data obeying (5) may be thought of as a generalization of such series solutions to an infinite class of such functional relations.

We plot below the $A=1=L, k=0$ case of $u$ for various $t$. The exponential initial data $u(x, 0)=e^{x}$ is shown as a black curve. With our sign conventions in the Euler-Monge equations, the wave moves to the left. For negative $t$ the solution is also unbounded as $x \rightarrow+\infty$, but non-singular for finite $x$, as shown by green curves in the right-most part of the Figure for various negative times. For positive $t$, vertical profiles occur where the curve has infinite slope, at the points indicated by small circles on the tips of the series-defined segments of $u(x, t)$ shown by green curves in the left-most part of the Figure for various positive times. The series fails to converge for larger values of $x$, beyond these tips. (In more realistic situations, this may be indicative of some missing physics, such as would be represented by adding higher $x$ derivatives in the original PDE, as in Burgers' equation, see [8].) However, the implicit definition of $u(x, t)$ given by the functional relation (5) serves to define upper branches of $u(x, t)$ that are shown by the orange curves extending beyond the location of the vertical face. These upper branches may be thought of as the overhanging portions of the breaking wave. A pretty numerical example of a continuous, integrable, piecewise exponential wave is obtained by joining rising and falling exponentials, so that the initial data is $\exp (-|x|)$. The time evolution of this example can be found graphically displayed on the website of one of the authors. (http://curtright.com/waves.html) 


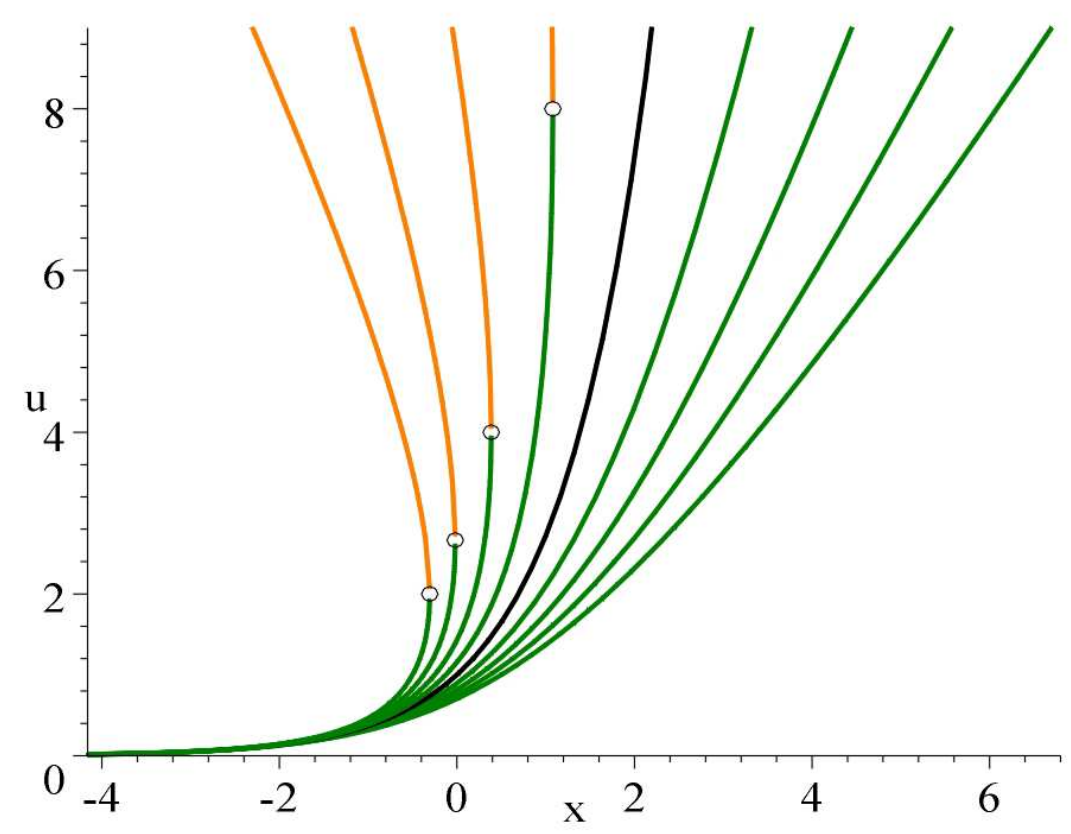

Evolution of the "Lambert front" for $t=-1 / 2$ to $+1 / 2$ in $\Delta t=1 / 8$ steps

The location of the vertical face moves to the left as $x(t)=-1-\ln t$, more slowly than the upper branch of the front, and reaches the origin at $t=1 / e$. (Recall the model is non-relativistic.)

Moreover, we may extend the solution (51) of the Euler-Monge equation stemming from the exponential initial data, using the analysis of the preceding subsections, to find the constant body-force solution arising from the same initial data.

$$
u(x, t)=k t-\frac{L}{t} W\left(-\frac{A t}{L} \exp \frac{1}{L}\left(x+\frac{1}{2} k t^{2}\right)\right) .
$$

We also find the corresponding linear body-force solution.

$$
u(x, t)=k x \tan k t-\frac{L k}{\sin k t} W\left(-\frac{A \tan k t}{L k} \exp \frac{x}{L \cos k t}\right) .
$$

Thus we have conditions for the wave profile to be vertical (signaling the local formation of shocks) whenever the arguments of the Lambert functions are equal to $-1 / e$, i.e.

$$
t_{\text {break }}=\frac{L}{A e} e^{-x / L}, \quad t_{\text {break }}=\frac{L}{A e} \exp \frac{-1}{L}\left(x+\frac{1}{2} k t_{\text {break }}^{2}\right), \quad \text { or } \tan k t_{\text {break }}=\frac{L k}{A e} \exp \left(\frac{-x}{L \cos k t_{\text {break }}}\right),
$$

respectively, for the three situations considered. The latter two conditions are very implicit determinations of $t_{\text {break }}$.

\section{Simple consequences of the extra dimension approach}

In this section a trivial method is given to construct an infinite number of solutions to the diffusion equation starting from a solution of the Monge equation, which might well be taken as one of the particular solutions of explicit form.

This follows very simply from the existence of operators which commute with the diffusion operator. Consider the case where $p=0$, i.e the free case. Then because $\frac{\partial}{\partial t}, \frac{\partial}{\partial a}, \frac{\partial}{\partial x}$ and the "boost" generator $x \frac{\partial}{\partial x}-a \frac{\partial}{\partial a}$ all commute with the operator $\frac{\partial}{\partial t}-\frac{\partial^{2}}{\partial x \partial a}$, we can find an infinite class of solutions of the diffusion equation based upon the solution $u$ of the Monge equation by operating upon the "primitive" solution $\mathbb{U}(x, t, a)$ with arbitrary functions of these operators. (In fact only three such operators are required, as the diffusion operator is itself a combination of the others.) For example, consider

$$
G\left(\frac{\partial}{\partial a}\right) \frac{\partial}{\partial x} \mathbb{U}(x, t, a)=G(u) \frac{\partial u}{\partial x} e^{a u(x, t)}
$$


where $G$ is an arbitrary function with no singularity at the origin, or

$$
G\left(\frac{\partial}{\partial a}\right) \frac{\partial}{\partial t} \mathbb{U}(x, t, a)=G(u) \frac{\partial u}{\partial t} e^{a u(x, t)} .
$$

Note that in fact this latter class is really the same as the former, on account of the differential equation satisfied by $u$ : All time derivatives may be replaced by derivatives with respect to $x$. In the case of generic $g(x) \neq 0$, the only operator which commutes with the diffusion operator (11) is $\frac{\partial}{\partial t}$. But there are exceptional cases. For example, when $g(x)=k^{2} x$, the boost generator $x \frac{\partial}{\partial x}-a \frac{\partial}{\partial a}$ also commutes.

Suppose $u$ is a particular solution of the Monge equation, in the case of the linear pressure-gradient term, where $g(x)=k^{2} x$, such as one of the solutions given above: $u=k x \tan (k t)$ or $u=-k x \cot (k t)$. Suppose further that another solution of the diffusion equation is sought of the form $\mathbb{U}(x, t, a)=v(x, t) e^{a u(x, t)}$. Then it may be verified that $v(x, t)$ satisfies the equation

$$
\frac{\partial v}{\partial t}-\frac{\partial(u v)}{\partial x}=0
$$

If $u$ is known explicitly then this equation can be integrated: In the case $u=k x \tan (k t), v=-1 / \cos (k t)$, and in the case $u=-k x \cot (k t), v=1 / \sin (k t)$.

\section{Schrödinger and Euler-Monge relations}

The diffusion equation may be interpreted as a Schrödinger equation, upon complexification. To be explicit, consider the time-dependent Schrödinger equation for a harmonic potential in one dimension.

$$
\frac{\partial \psi(x, t)}{\partial t}=i \frac{\partial^{2} \psi(x, t)}{\partial x^{2}}-\frac{1}{4} i k^{2} x^{2} \psi(x, t) .
$$

We can rewrite this without change of content, together with its complex conjugate (with $(x, a)$ both real) as

$$
\begin{aligned}
& \frac{\partial \psi(x+i a, t)}{\partial t}=\frac{\partial^{2}}{\partial x \partial a} \psi(x+i a, t)-\frac{1}{4} i k^{2}(x+i a)^{2} \psi(x+i a, t), \\
& \frac{\partial \bar{\psi}(x-i a, t)}{\partial t}=\frac{\partial^{2}}{\partial x \partial a} \bar{\psi}(x-i a, t)+\frac{1}{4} i k^{2}(x-i a)^{2} \bar{\psi}(x-i a, t) .
\end{aligned}
$$

Multiply the first by $\bar{\psi}(x-i a, t)$ and the second by $\psi(x+i a, t)$ and add to obtain

$$
\frac{\partial}{\partial t}(\psi(x+i a, t) \bar{\psi}(x-i a, t))=\frac{\partial^{2}}{\partial x \partial a}(\psi(x+i a, t) \bar{\psi}(x-i a, t))+k^{2} a x(\psi(x+i a, t) \bar{\psi}(x-i a, t)) .
$$

Defining the complex point-split density $\rho(x, t, a)=\psi(x+i a, t) \bar{\psi}(x-i a, t)$, we see that $\rho$ obeys a diffusion type equation which is solvable in terms of a one-dimensional Euler-Monge equation with a linear pressure-gradient term, in the manner indicated above. Defining as previously $x=\frac{1}{2}\left(x_{1}+i x_{2}\right), a=\frac{1}{2}\left(x_{1}-i x_{2}\right)$, we find that $\rho$ satisfies formally a two-dimensional diffusion equation with a complex harmonic potential:

$$
\frac{\partial \rho}{\partial t}=\frac{\partial^{2} \rho}{\partial x_{1}^{2}}+\frac{\partial^{2} \rho}{\partial x_{2}^{2}}-\frac{1}{4} i k^{2}\left(x_{1}^{2}+x_{2}^{2}\right) \rho .
$$

This transformation of the Schrödinger equation is subtle, however, as now the arguments of the original wave functions are complex for real $x_{1}$ and $x_{2}: \quad x \pm i a=\frac{1}{2}(1 \pm i)\left(x_{1} \pm x_{2}\right)$.

For real point-splitting, there are an infinite number of conserved charges and corresponding current densities for the free-particle Schrödinger equation, in an arbitrary number of spatial dimensions, as is well-known. These conservation laws follow immediately from

$$
\partial_{t} \psi(\mathbf{x}, t)=i \kappa \nabla^{2} \psi(\mathbf{x}, t), \quad \partial_{t} \bar{\psi}(\mathbf{x}, t)=-i \kappa \nabla^{2} \bar{\psi}(\mathbf{x}, t)
$$

with $\kappa=\frac{1}{2 m}$. For the real point-split probability density (just the density matrix in position-position representation),

$$
\rho(\mathbf{x}, t, \mathbf{a}) \equiv \bar{\psi}(\mathbf{x}-\mathbf{a}, t) \psi(\mathbf{x}+\mathbf{a}, t)
$$

it follows that

$$
\partial_{t} \rho(\mathbf{x}, t, \mathbf{a})=\nabla \cdot \mathbf{J}(\mathbf{x}, t, \mathbf{a})
$$




$$
\mathbf{J}(\mathbf{x}, t, \mathbf{a})=i \kappa \bar{\psi}(\mathbf{x}-\mathbf{a}, t)\left(\nabla_{\mathbf{x}} \psi(\mathbf{x}+\mathbf{a}, t)\right)-i \kappa\left(\nabla_{\mathbf{x}} \bar{\psi}(\mathbf{x}-\mathbf{a}, t)\right) \psi(\mathbf{x}+\mathbf{a}, t) .
$$

However, as above, it is more in line with 10 to write this current density as

$$
\mathbf{J}(\mathbf{x}, t, \mathbf{a})=i \kappa \nabla_{\mathbf{a}} \rho(\mathbf{x}, t, \mathbf{a})
$$

so that the infinite set of conservation laws become just the second-order equation

$$
\partial_{t} \rho(\mathbf{x}, t, \mathbf{a})=i \kappa\left(\nabla_{\mathbf{x}} \cdot \nabla_{\mathbf{a}}\right) \rho(\mathbf{x}, t, \mathbf{a}) .
$$

We recognize this as just the Fourier transform of the Wigner-Moyal equation [25, 26, 28]. With

$$
f(\mathbf{x}, t, \mathbf{p})=\int d^{n} a e^{2 i \mathbf{a} \cdot \mathbf{p}} \rho(\mathbf{x}, t, \mathbf{a}),
$$

and $H=\kappa p^{2}$, the free-particle Wigner-Moyal equation is given in terms of Groenewold's $\star$-product [27] as

$$
\begin{aligned}
-i \partial_{t} f(\mathbf{x}, t, \mathbf{p}) & =-2 i \kappa \mathbf{p} \cdot \nabla_{\mathbf{x}} f(\mathbf{x}, t, \mathbf{p})=[H, f(\mathbf{x}, t, \mathbf{p})]_{\star}=H \star f-f \star H, \\
H \star f & =\kappa\left(\mathbf{p}-\frac{1}{2} i \nabla_{\mathbf{x}}\right)^{2} f, \quad f \star H=\kappa\left(\mathbf{p}+\frac{1}{2} i \nabla_{\mathbf{x}}\right)^{2} f
\end{aligned}
$$

Take one a gradient of (70) to obtain

$$
\partial_{t} \mathbf{J}(\mathbf{x}, t, \mathbf{a})=i \kappa\left(\nabla_{\mathbf{x}} \cdot \nabla_{\mathbf{a}}\right) \mathbf{J}(\mathbf{x}, t, \mathbf{a})
$$

Or take any number of a gradients. This gives a set of totally symmetric tensors conserved in precisely the same way.

$$
T_{i j \cdots k}(\mathbf{x}, t, \mathbf{a}) \equiv \partial_{a_{i}} \partial_{a_{j}} \cdots \partial_{a_{k}} \rho(\mathbf{x}, t, \mathbf{a}), \quad \partial_{t} T_{i j \cdots k}(\mathbf{x}, t, \mathbf{a})=i \kappa\left(\nabla_{\mathbf{x}} \cdot \nabla_{\mathbf{a}}\right) T_{i j \cdots k}(\mathbf{x}, t, \mathbf{a}) .
$$

Of course, $\mathbf{x}$ derivatives also commute through $\partial_{t}-i \kappa\left(\nabla_{\mathbf{x}} \cdot \nabla_{\mathbf{a}}\right)$, so these may also be used to obtain conserved tensors, but as total $x$ derivatives, they are not quite as interesting. On the other hand, the symmetric generators

$$
M_{(i j)}=x_{i} \partial_{x_{j}}+x_{j} \partial_{x_{i}}-a_{i} \partial_{a_{j}}-a_{j} \partial_{a_{i}}
$$

also commute with $\nabla_{\mathbf{x}} \cdot \nabla_{\mathbf{a}}$, and may be used to construct other conserved densities.

It is amusing that the conservation laws (70) are exactly of the form as in the introductory remarks of [10, with complexification of the second-derivatives' coefficient. Unfortunately, if that coefficient is not purely imaginary, then the construction falters for real point-splittings. In other words, for real functions obeying the real diffusion equation this particular collection of conservation laws does not exist. Still, it is also amusing that one could indeed think of the point-splitting as introducing extra dimensions, and doing so by dimension-doubling. The wave functions involved in the currents are then just two distinct types of particular free wave functions in twice the number of spatial dimensions, namely $\psi(\mathbf{x}, t, \mathbf{a})=\psi(\mathbf{x}+\mathbf{a}, t)$ and $\bar{\psi}(\mathbf{x}, t, \mathbf{a})=\bar{\psi}(\mathbf{x}-\mathbf{a}, t)$. They each obey the extra-dimensional equations

$$
\partial_{t} \psi(\mathbf{x}, t, \mathbf{a})=i \frac{\kappa}{2}\left(\nabla_{\mathbf{x}}^{2}+\nabla_{\mathbf{a}}^{2}\right) \psi(\mathbf{x}, t, \mathbf{a}), \quad \partial_{t} \bar{\psi}(\mathbf{x}, t, \mathbf{a})=-i \frac{\kappa}{2}\left(\nabla_{\mathbf{x}}^{2}+\nabla_{\mathbf{a}}^{2}\right) \bar{\psi}(\mathbf{x}, t, \mathbf{a}) .
$$

There are other solutions of these free particle equations, of course, but as in [10] we need only make use of particular solutions to encode the conservation laws.

\section{Generalized Bateman equation}

In previous investigations 29, the Euler-Monge equation was used to obtain the prototypical Bateman equation 30. This section addresses the question of what happens when the pressure term is added, and gives solutions of the resulting modified Bateman equation.

First of all we notice that the equation

$$
\frac{\partial^{2} u}{\partial t^{2}}=\frac{\partial}{\partial x}\left(u \frac{\partial u}{\partial t}\right)
$$

holds whatever the pressure term is, provided it is dependent only upon $x$. This implies the existence of a scalar $\phi(x, t)$ such that

$$
\frac{\partial u}{\partial t}=\frac{\partial \phi}{\partial x}, \quad u \frac{\partial u}{\partial t}=\frac{\partial \phi}{\partial t} .
$$


That is,

$$
u=\frac{\partial \phi / \partial t}{\partial \phi / \partial x}
$$

This and (2) imply that $\phi$ satisfies a Bateman-like equation, in interaction with an external field:

$$
\left(\phi_{x}\right)^{2} \phi_{t t}-2 \phi_{x} \phi_{t} \phi_{t x}+\left(\phi_{t}\right)^{2} \phi_{x x}=\left(\phi_{x}\right)^{3} g(x) \text {. }
$$

Note that the RHS preserves homogeneity in $\phi$, but breaks homogeneity in $t$ and $x$, in general. In the particular case where $g(x)=k$, a constant, and $u=\frac{1}{t}\left(-x+\frac{k t^{2}}{2}\right), \phi$ is determined by (78) to be $\phi=\frac{1}{2 t^{2}}\left(x+\frac{k t^{2}}{2}\right)^{2}$. Since any function of $\phi$ will also satisfy the Bateman-like equation (as is clear from (179)), we might as well take the square root of this for verification of 80 .

If $F(\phi), G(\phi)$ are arbitrary differentiable functions of $\phi$, then the general solution of the Bateman-like equation when the pressure-gradient is constant is given by solving implicitly for $\phi$ the following equation:

$$
\left(x+\frac{k t^{2}}{2}\right) F(\phi)+t G(\phi)=\text { constant } .
$$

Direct calculation establishes this result. We find

$$
\phi_{x}=\frac{-F}{\left(x+\frac{k t^{2}}{2}\right) F^{\prime}+t G^{\prime}}, \quad \phi_{t}=\frac{k t F+G}{F} \phi_{x},
$$

and hence

$$
\begin{aligned}
& \phi_{x} \phi_{x t}=\phi_{t} \phi_{x x}+\frac{F G^{\prime}-G F^{\prime}}{F^{2}} \phi_{x}^{3}, \\
& \phi_{t} \phi_{x t}=\phi_{x} \phi_{t t}-\frac{F G^{\prime}-G F^{\prime}}{F^{2}} \phi_{x}^{2} \phi_{t}-k \phi_{x}^{2} .
\end{aligned}
$$

Substitution of these last two expressions into the Bateman-like equation (80) proves the claim. The implicit equation (81) gives the general solution to (80), for constant pressure-gradient, as it depends upon two independent arbitrary functions.

A similar result can be found in the case of a linear pressure-gradient term, $g(x)=k^{2} x$, which is the unique example that preserves homogeneity in $x$ for (80). Here, the general solution is given by the implicit solution for $\phi$ of the following equation:

$$
\frac{\sin k t}{x} F(\phi)+\frac{\cos k t}{x} G(\phi)=\text { constant } .
$$

Once again, direct calculation establishes this result. This is the second known case where a generalized form of the Bateman equation is integrable, the other being the so-called "two dimensional Born-Infeld equation" 31, 32.

More general pressure-gradients appear to be less tractable for the Bateman-like equation, even implicitly, without resorting to the Euler-Monge form of the equation. Note that the recipe which worked to obtain implicit solutions of the driven Euler-Monge equation for arbitrary $g(x)$ is not as useful for [80), since thinking of $t(x, \phi)$ instead of $\phi(x, t)$ does not linearize that equation.

\section{Summary}

We have considered here the effects of given pressure-gradients on the one-dimensional flows of the Euler-Monge equation, and on the corresponding driven diffusion/Schrödinger equations expressed in an extra dimension. The extra dimension method provides some additional insight and computational tools beyond those given by the method of characteristics and the technique of constructing implicit solutions, which we have discussed and compared. While we have touched on some elementary features of conservation laws for the free particle Schrödinger case, including their relation to the Wigner-Moyal equation, a full analysis of higher-dimensional driven flows along the lines of [10] remains to be given [33. The effects of viscosity have also not been considered. (For a recent study including viscosity effects, see [8].) We hope to return to these issues in a subsequent paper.

Acknowledgements: We thank the referee for raising the issue of shock fronts, and J Nearing for bringing to our attention P Lax's concise monograph on the subject. This research was supported in part by a Leverhulme Emeritus Fellowship and by NSF Award 0073390. 


\section{References}

[1] A Polyakov, "Turbulence without pressure", Phys Rev E52 (1995) 6183-6188 hep-th/9506189

[2] V P Dmitriyev, "Towards a Mechanical Analogy of a Quantum Particle: Turbulent Advection of a Fluid Discontinuity and Schroedinger Mechanics" Supplementary papers of the BSPS PIRT conference, London, Sept 1996, pp.68-73; Apeiron, vol. 7, no 3-4, pp. 161-172 (2000) physics/9904034

[3] O K Pashaev and Jyh-Hao Lee, "Self-Dual Vortices in Chern-Simons Hydrodynamics" Theor Math Phys 127 (2001) 779-788 hep-th/0104258

[4] M Hossein Partovi, "Hamilton-Jacobi Formulation of KS Entropy for Classical and Quantum Dynamics" quant-ph/0107083

[5] R Jackiw, S-Y Pi, and A P Polychronakos, "Noncommuting Gauge Fields as a Lagrange Fluid" Annals Phys 301 (2002) 157-173 hep-th/0206014; B Bistrovic, R Jackiw, H Li, V P Nair, S-Y Pi, "Non-Abelian Fluid Dynamics in Lagrangian Formulation" Phys Rev D67 (2003) 025013 hep-th/0210143

[6] P J Love and B M Boghosian, "Quaternionic Madelung Transformation and Non-Abelian Fluid Dynamics" hep-th/0210242

[7] D H Delphenich, "A Geometric Origin for the Madelung Potential" gr-qc/0211065

[8] S Boldyrev, T Linde, and A Polyakov, "Velocity and velocity-difference distributions in Burgers turbulence" nlin.CD/0305014

[9] E Madelung, "Eine anschauliche Deutung der Gleichung von Schrödinger" Naturwiss 14 (1926) 1004; "Quantentheorie in hydrodynamischer Form" Z Phys 40 (1926) 332.

[10] T L Curtright and D B Fairlie, "Extra Dimensions and Nonlinear Equations", J Math Phys 44 (2003) 2692-2703 math-ph/0207008

[11] L Euler, Opera Omnia, Series II, Opera mechanica et astronomica, Volumes 12 and 13, Commentationes mechanicae ad theoriam corporum fluidorum pertinentes, edited by Clifford Ambrose Truesdell, Birkhäuser, 1981.

[12] G Monge, Application de l'analyse a la géométrie, Bernard, 1807 (H. Perronneau).

[13] B Riemann "Uber die Fortpflanzung ebener Luftwellen von endlicher Schwingungsweite", Göttingen Abhandlunger, Vol. viii, p 43 (Werke, 2te Aufl., Leipzig, 1892, p 157).

[14] L Debnath, Nonlinear partial differential equations for scientists and engineers, Birkhäuser, 1997.

[15] B A Dubrovin and S P Novikov, "Hydrodynamics of weakly deformed soliton lattices. Differential geometry and Hamiltonian theory", Russian Math. Surveys 44 (1989) 35-124.

[16] G B Whitham, Linear and Nonlinear Waves, Wiley-Interscience, 1974.

[17] If $(x, a)$ are conjugate complex co-ordinates, $x=\frac{1}{2}\left(x_{1}+i x_{2}\right), a=\frac{1}{2}\left(x_{1}-i x_{2}\right)$, then recall that $\frac{\partial^{2}}{\partial x \partial a}=\frac{\partial^{2}}{\partial x_{1}^{2}}+\frac{\partial^{2}}{\partial x_{2}^{2}}$.

[18] M Abramowitz and I A Stegun, Handbook of Mathematical Functions, U S Government Printing Office, Washington D.C. http://dlmf.nist.gov/

[19] B Defacio, G J Johnson and M Lapidus, "Feynman's Operational Calculus and Evolution Equations" Acta Applicandae Mathematicae 47 (1997) 155-211.

[20] In this regard, note in (42) that $(\sin k t)^{j} \frac{d^{j}}{d x^{j}}=(\tan k t)^{j} \frac{d^{j}}{d(x / \cos k t)^{j}}$.

[21] This transformation is analogous to the relation between free point particle motion, with $x_{\text {free }}(t)=x_{0}+v_{0} t$, and simple harmonic motion, with $x_{\mathrm{SHO}}(t)=x_{0} \cos k t+\frac{1}{k} v_{0} \sin k t$, whose two forms are connected by a dynamical rescaling and substitution for the time variable: $x_{\mathrm{SHO}}(t) / \cos k t=x_{\text {free }}\left(t \rightarrow \frac{1}{k} \tan k t\right)$.

[22] As further illustration of this transformation technique, if $u(x, t)$ is a solution of the undriven equation (3), then $\mathfrak{u}(x, t) \equiv u(x+s(t), t)+d s(t) / d t$ satisfies $\partial_{t} \mathfrak{u}=\mathfrak{u} \partial_{x} \mathfrak{u}+d^{2} s(t) / d t^{2}$. 
[23] P D Lax, "Hyperbolic Systems of Conservation Laws and the Mathematical Theory of Shock Waves" SIAM Regional Conference Series in Applied Mathematics 11, 1973.

[24] R M Corless, G H Gonnet, D E Hare, D J Jeffrey, and D E Knuth, "On the Lambert W function" Adv Comp Math 5 (1996) 329-359. In Maple the function is called LambertW $(z)$, or, on its other branches, LambertW $(k, z)$. In Mathematica it is known as the ProductLog function. (See http://mathworld.wolfram.com/for a guide to the literature.)

[25] E Wigner, "On the Quantum Correction For Thermodynamic Equilibrium" Phys Rev 40 (1932) 749-759.

[26] J Moyal, "Quantum Mechanics As A Statistical Theory" Proc Camb Phil Soc 45 (1949) 99-124.

[27] H Groenewold, "On The Principles Of Elementary Quantum Mechanics" Physica 12 (1946) 405-460.

[28] C K Zachos, D B Fairlie, and T L Curtright, Quantum Mechanics in Phase Space, World Scientific Publishing Co., to appear.

[29] D B Fairlie, J Govaerts and A Morozov, "Universal Field Equations with Covariant Solutions" Nucl Phys B373 (1992) 214-232.

[30] H Bateman, "Some recent researches on the motion of fluids", Monthly Weather Rev. 43 (1915) 163-170.

[31] M Arik, F Neyzi, Y Nutku, P J Olver, J M Verosky, "Multi-Hamiltonian structure of the Born-Infeld equation" J Math Phys 30 (1989) 1338-1344.

[32] D B Fairlie and J A Mulvey, "Integrable Generalisations of the 2-dimensional Born Infeld Equation" J Phys A27 (1994) 1317-1324.

[33] However, it should be readily apparent that the higher-dimensional flows produced by the special cases of constant or linear pressure-gradients can be expressed in terms of undriven solutions, with the same interpretations and forms as given above following (29) and (42). 